\section{Health promotion and early detection of cancer in older adults: A practical approach}

\author{
By Margaret Muir, Marlene Greenberg, Susan Plante, \\ Margaret Fitch, Linda Levstein and Eva King
}

\begin{abstract}
Little is known about the cancer-related knowledge, attitudes and health behaviours of older adults ( $>55$ years), or about designing health promotion and early detection intenventions responsive to their learning needs. A collaborative project was established with the intent of designing an appropriate program for older adults. The initial work included a community needs assessment using focus groups, one-on-one interviews and self-report surveys. The use of key community contacts was effective in locating older adult subjects (>55 years) through pre-established linkages with agencies. Various ethnocultural groups, low income communities and isolated individuals, as well as other pre-established groups, were inchuded in this study. The needs assessment found that: age is not perceived as a cancer risk factor; transportation is a barrier to screening; fear inhibits people from being screened; physicians are viewed as both the main source of expert cancer knowledge and as the gatekeepers to screening; family and peers are the main source of support, ethnospecific groups have different information needs; and finally, that lifestyle suggestions can reduce the risk of cancer. The findings indicated that community health promotion programs for older adults will require multiple approaches with a combination of strategies in order to meet their learning needs.
\end{abstract}

\section{Introduction}

Age is a significant risk factor for cancer. Consequently more Canadians will be affected, given the increasing proportion of the country's aging population. It is therefore important to educate those at risk about the disease. Currently, little is known about the cancer-related knowledge, attitudes and health behaviours of older adults, or about designing health promotion and early detection interventions responsive to their specific needs (Cancer 2000 Expert Panel, 1992; Dellefield, 1988; Mettlin, 1991; Rimer, Jones, Wilson et al, 1991). Recently the Cancer 2000 Expert Panel on Cancer in the Disadvantaged (1992) cited an urgent need to investigate health promotion barriers, cancer risk reduction and early detection in older adults, and called for programs to be responsive to their needs. Dellefield (1988) cites obstacles to achieving cancer risk reduction and early detection of cancer for the older adults. These obstacles include certain health behaviours common to older adults, practices of some health care professionals, and inherent limitations in the health care system. Many older adults are not aware of the increased risk of developing cancer, neglecting to participate in screening programs and practise self-examination (Williams \& Dueker, 1985; Morra \& Blumberg, 1991). Changes are often viewed, by both consumers and health care professionals, as part of the aging process when in fact they are pathological (Satariano, Belle \& Swanson, 1986). Nurses and doctors may not be fully aware of the significant risk of developing cancer with increased age and may believe that aggressive intervention is not needed.

Typically, health promotion and screening programs have targeted the younger population, but older adults are now realizing that the changes they experience are perhaps due to lack of health-promoting practices in the past. Other factors affecting screening behaviours lie in the variations within this population in terms of income, accommodation, education, physical capacity, breadth of interest and ethnocultural background (Gray, 1985; Dellefield, 1988). To date, there is very little written on the prevention and early detection of cancer in older adults. The planning of health promotion and cancer programs for the older adult must be responsive to all of these issues and especially to gender, socioeconomic and ethnocultural issues (Given \& Given, 1989; White, Fishman, Guthrie \& Fagan, 1993). This article describes the approach taken in an urban setting to plan a health promotion and cancer program for older adults. The steps used and lessons learned could be of assistance to nurses wishing to implement a similar program.

While it is recognized that the increased risk applies to those over the age of 65 , health promotion as an intervention strategy more appropriately applies to those in the preceding decade. Therefore, older adults, for purposes of this project, were selected as individuals over the age of 55 years.

Fundamental to a successful program in the community is the involvement of the community in the formative stages. Hence, we included community representatives throughout the project.

The specific objectives of the project were:

- to establish a needs assessment working group that is community-based - to learn about the barriers to health promotion and early detection of cancer for the older adult in a diversified population

- to involve the community in the identification of health promotion needs and solutions

- to access and collaborate with existing community resources

- to form a program advisory group (PAG) which would identify program strategies to address the needs identified in the assessment, and

- to evaluate the effectiveness of project activities.

It is imperative to learn about a community and its needs at the onset if program planning is to be successful.

\section{Process}

The project process consisted of the following steps (See Figure One):

\section{The needs assessment working group (NAWG)}

The NAWG was formed with representatives from the Toronto-Sunnybrook Regional Cancer Centre, the North York Public Health Department, The Anne Johnson Health Station (a community health centre) and an older adult from a local community agency. The NAWG's primary role was to oversee the community needs assessment. The needs assessment had the following three purposes: 1) to build a data pool on older adults' knowledge, attitudes and behaviours about health promotion and early detection of cancer;

2) to discover the best method(s) for learning about these older adults' ideas and views; and

3) to determine appropriate strategies for these older adults to learn information in a program context.

A community outreach coordinator was hired by NA WG to conduct the needs assessment. She established and maintained contacts by building the trust of community residents and service providers. The coordinator also recorded the needs assessment data and evaluated the needs assessment process (with NAWG) on an ongoing basis.

\section{The community assessment}

To validate and expand on the initial project ideas, and to identify and determine how to access the population of interest, the NAWG held a one-time focus group (stakeholder group) with six older adults. This group session underscored the ease with which participants spoke about cancer, the value of a group approach and the interest older adults had in health.

Given that the catchment area was very large, four smaller communities within the catchment area were selected for the purposes of the community needs assessment. Each community had a high concentration of older adults with different incomes, cultures and linkages to the community.

Margaret Muir, RN, MHSc, is with the Health Information Centre, Sunnybrook Health Science Centre (formerly with the Anne Johnson Health Station); Marlene Greenberg, MS, and Margaret Fitch, RN, PhD are at Toronto-Sunnybrook Health Science Centre; Susan Plante, RN, MSN, is with North York Public Health Department; Linda Levstein was the community outreach coordinator; and Eva King was a community representative. 
Contact was made initially with the target communities through the existing networks of the NA WG members. These contacts included seniors' apartment buildings, seniors' centres, public health, ethnocultural organizations, community health centres and informal community leaders.

Key informants, service providers, local residents and published reports provided information which characterized each community and its members. This included each community's physical structure, socioeconomic status, ethnicity, family structure, barriers encountered in obtaining health care and existing health services available for older adults.

\section{A multi-method community needs assessment approach}

The three methods used in this community needs assessment were: focus groups, interviews and a self-report survey. Each will be described briefly. The actual findings from each method are reported by Fitch, Greenberg, Levstein et al. (1996).

\section{The focus group}

Ten focus groups were held with a total of 158 older adults. Six of the focus group sessions were held with existing groups who meet regularly in seniors' settings. These groups were held with the singular purpose of accessing the opinions of older adults. The last four focus groups were formed and held specifically for the purpose of this project; to access different cultural groups as the issue of culture emerged as a factor in ideas about health. The specific cultural focus included Italian, Greek, Spanish and Chinese groups and required more effort to access than did the others. The focus groups required about an hour and a half to complete, with the exception of the four focus groups conducted through an interpreter. The focus groups were co-facilitated with an older adult.

The specific topics addressed during the focus group sessions included

Figure One: Project activity flow

Forming the needs assessment working group (NAWG)

- Defined goals and objectives and terms of reference

- Identified the type of information to collect

- Facilitated the needs assessment

\section{Stakeholder focus group}

- Validated initial ideas and decisions with the members of population of interest

- Determined the types of questions to ask

- Explored the feasibility of using a focus group format with older adults

Defining the role of community outreach coordinator - Developed job description

- Hired community outreach coordinator

\section{Conducting the community assessment}

- Profiling: learning about the communities

- Networking: establishing community contacts

- Needs assessment (focus group, interviews, surveys)

\section{Forming the program advisory group (PAG)}

- Assessed needs assessment data

- Made recommendations for designing community health promotion program around prevention and early detection of cancer in older adults the older adults' knowledge, beliefs, attitudes, behaviours, barriers and strategies related to cancer prevention and early detection regarding Pap smears, mammograms, testicular, prostate, skin and bowel/colon cancers. The focus groups offered rich indepth discussion of these topics.

\section{The interview}

Nine individuals were interviewed, three of whom were isolated and six active. The interviews were conducted in the interviewees' homes and lasted approximately 60 minutes. An interpreter was used twice.

Individual interviews were conducted to determine if there were differences in the amount and kinds of information disclosed in the individual interview compared to the group setting. One-on-one interviews were also used to reach isolated seniors. Interviewees were selected to represent the range of economic status and ethnicity of the community at large. These individuals were identified through local community agencies. The same questions used for the focus groups were asked in the interviews.

\section{The survey}

It was seen as desirable to find an easily administered method to assess older adults' knowledge about cancer and cancer screening. The survey strategy was a "pen and paper" approach. NAWG's recommendations were to reach a large sample of the target population, to determine the feasibility of gathering data using this method with older adults, and to see whether or not the data were different in the areas of knowledge and personal practices from the data gathered in the focus groups and interviews. The literature was searched to find an instrument which was short and easy to use. NAWG recommended the survey developed by Kane-Williams and White (1983) because it addressed issues similar to those covered in the focus groups and interviews. The survey, previously used with older adults, was selected based on the following criteria: addressed more than one type of cancer and cancer site, simple and short, contained true/false and/or multiple choice questions, easy to administer, addressed cancer knowledge and practice issues and had been tested for reliability and validity.

Demographic questions were added and the survey was translated into the particular languages reflective of the community (Italian, Greek, Punjabi, Hebrew, Russian, Chinese, Spanish). In order to ensure proper translation, the survey was translated back into English by another individual. The surveys were distributed through community workers via the community outreach coordinator. This was an efficient and cost-effective method. A small incentive was offered with each survey. This resulted in a very favourable response. Of the 783 surveys distributed, 588 were returned.

\section{Lessons learned}

Several lessons emerged from this early part of the project. Firstly, the areas of uncertainty identified regarding cancer prevention and early detection were similar in the focus groups, interview and survey findings. The focus groups added a dimension of rich exchange and support to the data collection which was not achieved with the one-to-one interviews. However, the interviews provided an avenue for more personal sharing. The survey was reported as easy for individuals to complete. The use of surveys in various languages was successful in collecting data. The survey could be used as a tool to measure cancer-related knowledge in future research and in other communities. The use of various data-gathering methodologies resulted in a great amount of data which added to the completeness of data, the complexity of data analysis and the richness of the findings.

Secondly, the data revealed that older adults have gaps and misconceptions in their knowledge about cancer and cancer prevention, but many were eager to learn. While age is considered a cancer risk factor, it was not perceived as such by a large majority of the participants. Many would turn to their doctors for answers about cancer, yet others felt doctors did not ask them about screening during the regular physician visits. Many felt fear inhibits people from being screened; they would turn to family and friends for reassurance. Men were more reluctant to discuss cancer and admit fear than women. Members of ethnospecific groups stressed that they had different information needs. They also requested materials and sessions in their own language and suggested the use of visual information.

Thirdly, the input from the older adult community itself about how to conduct the data collection was invaluable. Their comments include such ideas as: 
1) gathering in a group is a social event and refreshments are an important aspect of social events;

2) holding a focus group where older adults are, not expecting them to travel to you, is an important consideration;

3 ) including a small gift with the request to complete a survey is a "friendly" thing to do;

4) personal invitations to group sessions will be more effective than flyers/posters;

5) the views of isolated older adults are important and should be captured.

Fourthly, working collaboratively with several organizations set the stage for a productive project. Members from each of the organizations brought different skills and knowledge base. By each organization contributing different aspects to support the project (i.e. mailing, photocopying, secretarial support, meeting space, data analysis, etc.) no one organization carried the entire responsibility for the project.

Finally, it takes time to gain access to and develop trust in the ethnocultural and lower income communities, thus it is beneficial to use existing contacts. In the final analysis it was worth the extra time to identify and understand the issues in these communities.

\section{Feedback of results}

Once the community needs assessment had been completed, community members and organizations were approached to participate in a program advisory group (PAG). This group included older adults and service providers working in the cancer arena or with older adults. PAG served as the vehicle to feed the results of the needs assessment to the community and to ensure NAWG's recommendations were relevant. PAG's main suggestions after they reviewed all the assessment data were presented as strategies to reach older adults.

The recommendations included:

- relevant community organizations work in partnership to foster participation and mobilization of older adults;

- messages be developed which are positive, hopeful, entertaining and consistent in order to overcome the fear older adults perceive;

- provide information about how and when to seek screening to reduce reliance on the doctor who is the perceived gatekeeper;

- men's issues be addressed separately;

- older adults link with peers because of the importance of reassurance; - translated materials, visual information and key stakeholders from the population of interest be utilized in order to access ethnic groups; - isolated older adults be reached and involved through existing programs (e.g. Meals on Wheels, homemaking services) to overcome the transportation barrier;

- a celebrity spokesperson, special events and the media be used to deliver cancer messages in a positive and captivating way.

\section{Conclusion}

This project had as its goal to plan a cancer prevention and early detection program which was responsive to the needs of older adults. Before that could happen, information about the cancer-related knowledge, attitudes and health behaviours of older adults and the possible health promotion and early detection interventions which would be responsive to their specific needs had to be gathered. It is clear from the community needs assessment, the PAG and the older adults studied, that there is more work to be done. Clearly there is a lack of knowledge regarding cancer prevention and early detection in older adults.

The use of key community contacts facilitated a timely needs assessment which yielded depth and breadth of the information obtained. The inclusion of ethnocultural groups required extra time and planning to arrange translation and interpreters. This effort was rewarded by the documentation of a greater understanding of the issues of these communities. The inclusion of low income communities also required extra effort, yet similarly their issues became clearer.

The ongoing feedback of results to community members was important to validate the interpretation of the community's needs and resultant recommendations. NAWG's and PAG's feedback to the population of interest and relevant community members was valuable and this kind of feedback is recommended for similar projects in the future. The next phase of this work entails further contact with older adults and relevant community organizations so that they may select action strategies most appropriate for their own communities.

The group's work to understand the community underscored that the effective implementation of community health promotion programs for older adults requires many approaches, a combination of strategies and reinforcement in diverse community settings. Specifically, older adults should take an active role in their programming, building on their existing knowledge, skills and resources. The material should be presented in a light-hearted manner to relieve anxiety and the importance of sharing with peers should be stressed. Through these methods, older adults can be assisted to modify their lifestyles and engage in screening practices.

Nurses are in an important position to engage in health promotion, cancer prevention and early detection education. Whether engaging in individual nurse-to-patient interaction or speaking with a group of individuals, emphasis can be placed on understanding age is a risk factor and early detection is valuable. Many of the suggestions in this article could be used by nurses to plan programs for older adults. The overall process used could also be applied in other settings to mobilize community-based activity for cancer prevention and early detection.

\section{Acknowledgements}

Thank you to Maureen Cava and Natalia Krawetz for reviewing the manuscript. This project has received a grant from the Ontario Ministry of Health, Health Promotion Branch. Please note: Eva King passed away prior to the end of this project and writing of this mamuscript.

\section{References}

Cancer 2000 Expert Panel. (1992). Cancer and the Disadvantaged. Toronto: Canadian Cancer Society.

Dellefield, M.E. (1988). Informational Needs and A pproaches for Early Cancer Detection in the Elderly. Seminars in Oncology Nursing; 4(3), 156-168.

Fitch, M., Greenberg, M., Levstein, L., Muir, M., Plante, S., King, E. (1996). Health Promotion and Early Detection of Cancer in Older Adults: Needs Assessment for Program Development. (submitted)

Fitch, M., Greenberg, M., Levstein, L., Muir, M., Plante, S., King, E. (1996). Health Promotion and Early Detection of Cancer in Older Adults: Assessing Knowledge About Cancer. (submitted)

Given, B. and Given, C.W. (1989). Cancer Nursing for the Elderly - A Target for Research. Cancer Nursing; 12(2):71-77.

Gray, J.A. (1985). Education for Health in Old Age. Prevention of Disease in the Elderly. London: Churchill Livingstone: 200-230.

Kane-Williams, E., White, J.E. (1983). Community-based cancer education for the elderly. Progress in Cancer Control IV: Research in the Cancer Centre. 113-122.

Mettlin, C., Bonfiglio, J., Berg, R.L., Nelson, G.R., Patterson, W.B., Richardson, J., Runer, B., Sorenson, A., Warnecke, R. (1991). Prevention and Detection in Older Persons. Cancer; 68; 2530-2533.

Morra, M.E., Blumberg, B.D. (1991). Women's Perceptions of Early Detection in Breast Cancer: How Are We Doing? Seminars in Oncology Nursing; 7(3):157-160.

Satariano, W.A., Belle, S.H., Swanson, G.M. (1986). The Severity of Breast Cancer at Diagnosis: A Comparison of Age and Extent of Disease in Black and White Women. American Journal of Public Health; 76(7):779-782.

Rimer, B., Jones, W.L., Wilson, C., et al. (1983). Cancer in the elderly: A cancer control challenge, in Mettlin, C., Murphy, G.P. Eds., Progress in Cancer Control IV: Research in the Cancer Centre. New York: Alan R. Liss.

White, J.E., Fishman, N.W., Guthrie, B. Fagan, L.K.(1993). Increasing Cervical Cancer Screening Among Minority Elderly. Journal of Gerontological Nursing. May: 28-34.

Williams, G.O., Dueker, D.L. (1985). The Non-Use of Free Health Screening by Rural Elderly. American Journal of Preventive Medicine; 1(4):52-57. 